\title{
Formación en investigación y supervisión en programas de doctorados
}

Research Training and Supervision in Doctoral Programs

Formation pour la recherche et supervision aux programmes de doctorat

Formação em pesquisa e supervisão em programas de doutorados

Fecha de recepción: 8 DE ENERO DE 2016/Fecha de aceptación: 25 DE JULIO DE 2017/Fecha de disponibilidad en línea: 1 DE DICIEMBRE DE 2017

Encuentre este artículo en http://magisinvestigacioneducacion.javeriana.edu.co/

Escrito por Alejandro Proestakis-Maturana Universidad Católica del Norte Antofagasta, Chile Aproesta@ucn.cl

Walter Terrazas-NúÑez Universidad Católica del Norte Antofagasta, Chile wterraza@ucn.cl

\section{Resumen}

El aumento en oferta y matrículas de programas de doctorado es un fenómeno global que demanda atención directa de aquellos involucrados. Esta investigación tuvo por objetivo comprender las percepciones que tiene la Universidad, sobre los procesos de formación en investigación y la supervisión en programas de doctorado. Se interpretaron los discursos de directores, supervisores y estudiantes de programas de doctorado. Los principales resultados son que la formación en investigación y los procesos de supervisión tienen un fuerte componente motivacional y emocional, y la existencia de normas explícitas e implícitas del contexto científico que permean la formación de los futuros investigadores.

\section{Palabras clave}

Educación universitaria; posgrado; actividad de investigación; investigador; investigación

Para citar este artículo / To cite this article / Pour citer cet article / Para citar este artigo 
Keywords

Higher education; postgraduate;

research activity; researcher; research

\section{Abstract}

The increasing offer and enrollment of doctoral programs is a globalized phenomenon that demands direct attention from those involved. This article aimed to understand the perceptions that the University has on the processes of research training and supervision in doctoral programs. Discourses of Directors, Supervisors and students of doctoral programs were interpreted. Results showed that research training and supervisory processes have a strong motivational and emotional component, and the existence of explicit and implicit rules of the scientific context that permeate the education of future researchers.

\section{Mots clés}

Éducation universitaire; master; activité de recherche; recherche

\section{Résumé}

L'augmentation de l'offre d'inscriptions de programmes de doctorat est un phénomène globalisé qui demande l'attention directe de ceux qui y sont impliqués. Cet article de recherche a eu en tant qu'objectif comprendre les perceptions qui a l'Université; par rapport aux processus de formation pour la recherche et la supervision aux programmes de doctorat. On a interprété les discours de Directeurs, Inspecteurs et étudiants de programmes de doctorat. On a obtenu en tant que principaux résultats, que la formation pour la recherche et les processus de supervision ont un fort composant motivationnel et émotionnel, et l'existence de normes explicites et implicites du domaine scientifique contribuent dans la formation des futurs chercheurs.

\section{Palavras-chave}

Educação universitária; pós-graduação; atividade de pesquisa; pesquisador; pesquisa

\section{Resumo}

O aumento em oferta e matrículas de programas de doutorado é um fenômeno global que demanda atenção direta dos envolvidos. Este artigo de pesquisa teve por objetivo compreender as percepções que tem a universidade sobre os processos de formação em pesquisa e a supervisão em programas de doutorado. Interpretaram-se os discursos de diretores, supervisores e estudantes de programas de doutorado. Obtém-se como principais resultados que a formação em pesquisa e os processos de supervisão têm um forte componente motivacional e emocional, e que existem normas explícitas e implícitas do contexto científico que permeiam a formação dos futuros investigadores. 


\section{Introducción}

Los programas de doctorado se han convertido en la herramienta central para entrenar a futuros investigadores, y alcanzar nuevos conocimientos y avances científicos, tecnológicos y económicos de un país (Gaeta, 2013). En este escenario, la formación de posgrado constituye un espacio privilegiado, pues permite desarrollar las competencias requeridas, con el fin de abordar problemas que requieren un enfoque conceptual más específico y efectivo (Kärner \& Puura, 2008). Así, la formación de futuros doctores se visualiza de forma sistemática, para ofrecer al estudiante una formación académica y científica que le permita introducirse en el mundo de la investigación, pero sobre todo fomentar el desarrollo del pensamiento lógico y científico (Krauskopf, 2003). Esto le permitiría al estudiante generar investigación de acuerdo a los estándares científicos esperados y a las demandas que el entorno pueda ofrecer (Kleijn, Mainhard, Meijer, Pilot \& Brekelmans, 2010).

En la actualidad, hay un aumento progresivo y significativo tanto en número de programas como en las matrículas. Estados Unidos, Europa y América Latina presentan tasas de aumento durante los últimos cuarenta años en la oferta de estos programas, cercanas al 60\% y matrículas que rodean cifras similares (Kehm, 2006; OECD, 2013; Sadlak, 2004). Sin embargo, las tasas de deserción y compleción crecen a la par (Council of Graduate Schools, CGS, 2010; Litalien \& Guay, 2015; Nettles \& Millett, 2006).

Este incremento en la oferta, matrícula y deserción, junto a los cambios en las demandas de investigación del entorno, ha puesto especial presión a la práctica de supervisión para cambiar, flexibilizar y adaptarse a estas nuevas condiciones (Edwards, 2002; Pearson \& Kayrooz, 2004).

En este contexto, experiencias como la europea y la estadounidense han puesto especial atención en este fenómeno, específicamente, en cómo certificar la calidad educativa de los programas de doctorado (Sadlak, 2004). Sin embargo, consecuencias residuales de esta situación, como el elevado aumento en los tiempos de compleción y deserción en estos, parecen no tener la atención necesaria de los medios y la comunidad científica (Litalien \& Guay, 2015).

Las investigaciones en torno a este tema concuerdan en que si bien el abandono en los programas de doctorado es de naturaleza multidimensional (Gardner, 2009; McAlpine \& Norton, 2006), un mal proceso de supervisión podría influir directamente en esto (Armstrong, Allinson \& Hayes, 2004; Bruce \& Stoodley, 2013; CGS, 2010; Gaeta, 2013; Kiley, 2011; Nulty, Kiley \& Meyers, 2009; Titus \& Ballou, 2014; Wright, Murray \& Geale, 2007).

Ahora bien, actualmente, el proceso de supervisión como concepto de investigación puede ser visto en dos vertientes; por un lado, como un proceso educativo y por otro, como la preparación formativa para la investigación (Boud \& Lee, 2008; Brew, 2001; Bruce \& Stoodley, 2013; Bruce, Stoodley \& Pham, 2009; Green \& Lee, 1999; Kiley \& Wisker, 2009; Manathunga, Lant \& Mellick, 2006). En este sentido, concebir la supervisión como un proceso educativo no solo permite reconocer al estudiante como un aprendiz en los procesos de investigación y futuro contribuyente a la comunidad científica (Bruce \& Stoodley, 2013), sino también validarla como una dinámica de interacción educativa, en la cual los factores y elementos explícitos e implícitos en ella toman mayor relevancia.

Una investigación llevada a cabo por Christine Bruce e lan Stoodley (2013) a un grupo de supervisores, permitió definir nueve categorías asociadas a la supervisión como espacio educativo. Las primeras tres categorías refieren a la supervisión desde la experticia del supervisor como pieza

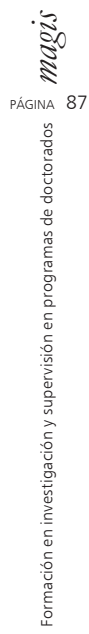

Descripción del artículo | Article description | Description de l'article | Artigo descrição

En este artículo de investigación, los autores hacen un análisis interpretativo sobre la formación en investigación y los procesos de supervisión en programas de doctorados de una universidad. Desde los procesos investigativos y la experiencia de los supervisores y estudiantes de posgrado, reflexionan sobre la importancia de los procesos de supervisión, las concepciones de investigación y aquellos elementos insertos en esta dinámica. Este artículo es resultado de la tesis Formación en investigación y supervisión en programas de doctorados; estudio de caso en los programas de doctorado de la Universidad Católica del Norte, para optar al grado de magíster en psicología social. 
central para el estudiante: promover el desarrollo de la investigación del supervisor; impartir conocimiento académico; y la defensa de los estándares académicos. Un segundo grupo centrado en la figura del supervisado: promover el aprendizaje de la investigación; basarse en la experiencia del estudiante; y fomentar el desarrollo de los estudiantes. Finalmente, un tercer grupo centrado en la comunidad y su influencia en el proceso de investigación: aventurarse en un territorio inexplorado; formar comunidades productivas; y la contribución a la sociedad.

Así, el proceso de supervisión y la relación existente entre supervisor y supervisado ocurren dentro de un contexto nacional e institucional (Nulty, Kiley \& Meyers, 2009). Es decir, es un proceso dinámico entre dos personas, sometido a reglas explícitas e implícitas del contexto en el cual se sitúan. Ejemplo de esto es lo propuesto por Susan K. Gardner (2009), Lynn McAlpine y Judith Norton (2006), que han sugerido un modelo integrativo tridimensional, que busca comprender la interacción e influencia entre los factores involucrados en la supervisión como espacio educativo, y su relación con el desgaste y abandono en los programas de doctorado; estos factores son sociales, institucionales y académicos.

El contexto social comprende las tendencias sociales, económicas y políticas que crean un ambiente dinámico, en constante cambio para las instituciones y para las personas que forman parte de ellas; el contexto institucional incluye las políticas universitarias que orientan los programas de posgrado; y finalmente, el académico, que contempla las reglas explícitas e implícitas relacionadas con la producción y comunicación del conocimiento, y la definición de roles tanto para supervisor como para supervisado.

Otros estudios — como los llevados a cabo por Margot Pearson y Carole Kayrooz (2004) - dan cuenta de cuatro factores involucrados en las prácticas de supervisión: progreso de la candidatura, mentoría, desarrollo del proyecto de investigación, y patrocinio al estudiante en la participación académica y la práctica profesional. Por otro lado, Renske A. M. Kleijn, M. Tim Mainhard, Paulien C. Meijer, Albert Pilot \& Mieke Brekelmans (2010) investigaron sobre la percepción de la relación entre supervisor y estudiante, y concluyeron entre otros resultados, la importancia de que los supervisores sean percibidos como altamente cercanos a sus estudiantes.

Al momento de investigar sobre los procesos de supervisión, es necesario considerar la variada gama de roles y características existentes para las figuras de los supervisores (Nulty, Kiley \& Meyers, 2009). Estudios como los de Christine Bruce e lan Stoodley (2013), Ross Deuchar (2008), Margaret Kiley (2011), Liina Lepp, Marvi Remmik, Mari Karm y Äli Leijen (2013), Margot Pearson y Carole Kayrooz (2004) y Stan Taylor y Nigel Beasley (2005) dan cuenta de esto y relevan la figura del supervisor como agente esencial en los procesos educativos de los futuros investigadores.

Sin embargo, si bien las características, roles y tipos de supervisores influyen en los procesos de supervisión y sus resultados, las creencias de los propios supervisores sobre el proceso en sí los hacen más competentes a la hora de formar a los nuevos investigadores (Wright, Murray \& Geale, 2007).

Actualmente, las universidades están viviendo un fenómeno no menor. Algunos autores lo denominan comercialización de la educación de investigadores en programas de posgrados, o en otras palabras, la focalización en producción investigativa como índice de calidad universitaria (Brooks \& Heiland, 2007; Deem, 2006). Esto toma mayor relevancia al visualizar el espacio de formación de futuros investigadores como una aventura solitaria entre supervisor y supervisado (Gunnarsson, Jonasson \& 
Billhult, 2013). Por un lado, se evalúan implícita y explícitamente la experticia del investigador y, por otro, se le pide tomar el rol de formador de los nuevos investigadores (Deuchar, 2008).

Entonces, comprender cómo los involucrados en este proceso significan, entienden y viven la formación en investigación, se vuelve esencial para plantear líneas de trabajo y mejora en las propias universidades. Esto permitirá conocer y comprender las concepciones existentes en la universidad sobre la formación científica y los procesos de supervisión en programas de doctorado. Este fenómeno nos permite reflexionar sobre las prácticas académicas explícitas e implícitas de cada cultura académica, y su injerencia en la formación de futuros investigadores. Esa reflexión — de acuerdo a Tony Becher (1993) y Brian Edwards (2002) — puede demandar grandes cambios estructurales en la cultura organizacional y académica establecida de las universidades.

Se tomará como estudio de caso la Universidad Católica del Norte, UCN, de la ciudad de Antofagasta, Chile. Esta institución de derecho público, fundada el 31 de mayo de 1956, tiene alrededor de 12.000 estudiantes. Posee tres sedes emplazadas en el norte de Chile, ubicación que ha marcado su quehacer pues la UCN, desde sus orígenes ha mantenido su vinculación con el desarrollo regional del norte de Chile. Actualmente, cuenta con 43 programas de pregrado y 36 de posgrado, de los cuales 28 corresponden a magíster y 8 a doctorados.

Su proyecto educativo institucional se fundamenta en tres pilares: un modelo pedagógico centrado en el sujeto que aprende con énfasis en el desarrollo de competencias para el futuro desempeño laboral; la formación en valores con énfasis en la reflexión ética sobre el ejercicio profesional; y la formación para la globalización con énfasis en el desarrollo de destrezas para el uso de recursos tecnológicos y el aprendizaje de un segundo idioma. Así mismo, la creación y el fortalecimiento de los programas de doctorado son otras acciones estratégicas centrales en la UCN con los que se busca contribuir a la generación y difusión de conocimiento, formar capital avanzado, al integrar las necesidades científicas y tecnológicas de la zona geográfica en que se emplaza la UCN y las capacidades de investigación institucionales.

\section{Metodología}

Se ha optado por el estudio de caso, ya que este nos permite conocer a profundidad el fenómeno investigado, y descubrir posibles líneas de estudios e investigación no visualizadas previamente (Berg, 2001).

La unidad de análisis de esta investigación está compuesta por los tres programas de doctorado de la Universidad Católica del Norte, que se imparten actualmente en la sede Antofagasta. Bajo la tipología propuesta por Tony Becher (1993), se diferenciaron de la siguiente forma: doctorado en ciencias, mención geología, como ciencia dura/aplicada; doctorado en ciencias, mención matemáticas, como dura/pura; y el doctorado en psicología, como blanda/aplicada. Para esta investigación, la tipología blanda/pura no fue incorporada, debido a la inexistencia de un programa de doctorado afín.

Todos los programas tienen una estructura similar con énfasis en formación para la investigación, con una duración de ocho semestres y el inicio del trabajo de tesis desde el segundo año. El principal objetivo del doctorado en ciencias mención geología, desde 2001, es la formación de científicos autónomos y creativos que formen o integren equipos de trabajo en áreas de investigación básica y aplicada. El doctorado en matemáticas 
existe desde 2003 y su principal objetivo es la formación de investigadores profesionales en matemáticas que sean capaces de realizar investigación científica, original y de alto nivel como actividad permanente, así como generar proyectos de investigación y de desarrollo. El doctorado en psicología existe desde 2015 y su principal objetivo es la formación de investigadores y académicos expertos, autónomos en la generación y divulgación de conocimiento científico, con altos estándares de calidad, en el contexto de la zona centro-sur andina.

Se realizaron 18 entrevistas semiestructuradas. Una entrevista a una representante de la dirección de posgrado; por cada programa, se entrevistó a sus directores, quienes también cumplían el rol de supervisor en ellos; seis entrevistas a supervisores, 2 por cada uno de los programas quienes fueron recomendados por los directores; 8 estudiantes recomendados por los supervisores, de los cuales 2 pertenecían al programa de psicología; 4 al programa de geología y 2 del programa de matemáticas. El detalle y sexo de cada uno de ellos se describen en la tabla 1. La mayoría de la población entrevistada egresó de carreras de pregrado de la misma universidad, a excepción de dos de ellos.

Tabla 1

Unidad de análisis de programas de doctorado

\begin{tabular}{llll}
\hline Dirección de posgrado & Doctorado en matemáticas & Doctorado en psicología & Doctorado en geología \\
\hline $\begin{array}{l}\text { Representante de la dirección } \\
\text { de posgrado, RDP }\end{array}$ & Directora del programa & Director del programa & Director del programa \\
\hline & Supervisor & Supervisora & Supervisor \\
\hline Supervisor & Supervisor & Supervisor \\
\hline Estudiante $\mathrm{F}^{*} 4^{\circ}$ año $\mathrm{E}^{* *}$ & Estudiante M iniciando & Estudiante M $2^{\circ}$ año \\
\hline Estudiante $\mathrm{M}^{*} 4^{\circ}$ año $\mathrm{E}^{* *}$ & Estudiante M iniciando & Estudiante M $2^{\circ}$ año \\
\hline & & Estudiante $\mathrm{F} 2^{\circ}$ año \\
\hline \hline
\end{tabular}

* La tipología F y M, que corresponde a Femenino y Masculino, hace alusión al sexo de los estudiantes. Para los otros participantes, se utilizó la denominación en masculino o femenino de cada uno de ellos.

** La tipología E hace referencia a estudiantes extranjeros que cursan programas de doctorado en la Universidad Católica del Norte.

Fuente: elaboración propia

Para resguardar el anonimato de los participantes, se optó por la codificación referida en la tabla 2 al momento de reflejar los discursos en los resultados.

Tabla 2

Codificación de los participantes

\begin{tabular}{l|l}
\hline Participante & Codificación \\
\hline Supervisores & S1, S2, S3, S4, S5, S6* \\
\hline Directores de programa & D1, D2, D3* \\
\hline Estudiantes & E1, E2, E3, E4, E5, E6, E7, E8* \\
\hline Representante de la dirección de posgrado & RDP \\
\hline \hline
\end{tabular}

Para esta investigación, no se hará diferencia en el discurso por sexo o tipo de programa, a no ser estrictamente necesario. Fuente: elaboración propia 
El guion de preguntas se construyó con base en los supuestos teóricos revisados en esta investigación y a inquietudes de los investigadores en torno a la temática. Con el fin de comprobar la estructura del guion, evaluar su pertinencia y visualizar posibles preguntas que aportaran a esta investigación, se realizaron dos entrevistas con el guion propuesto a directores de programas de doctorado de una universidad en Colombia: un director de programa de ciencias e ingeniería, y una directora de un programa de ciencias sociales. Producto de esta instancia, se modificaron e incluyeron preguntas e ideas no contempladas previamente en el guion temático; se constituyó la secuencia del guion en temáticas como percepción sobre investigación, perfil del investigador/supervisor formación de investigadores, programas de posgrado, y elementos asociados en estos procesos.

El proceso de recolección de información se realizó entre mayo y septiembre de 2015. Se identificaron y contactaron los directores de cada uno de los programas, para luego comenzar con las entrevistas de supervisores y estudiantes. La elección de supervisores y estudiantes estuvo centrada en la recomendación tanto de directores, como de supervisores, y en su disponibilidad para realizar las entrevistas.

\section{Análisis de datos}

Los discursos de los participantes fueron estudiados mediante el análisis de contenido de acuerdo a lo planteado por Margrit Schreier (2012). Este constó de tres momentos: en primera instancia se leyeron todas las transcripciones de las entrevistas ( $N=18$ ), de esta forma se obtuvo una imagen general de la información y se tomó nota de los elementos emergentes (Braun \& Clarke, 2006). Luego, todos los discursos fueron pareados desde un análisis deductivo en dos categorías principales: (1) concepciones sobre investigación y (2) supervisión en programas de doctorado.

En una segunda fase, se buscó identificar y construir subcategorías claves, tanto desde un proceso deductivo al contrastar con elementos teóricos, como desde la emergencia de categorías desde un proceso inductivo de los datos. En la tabla 3, se evidencian dos grandes categorías; por un lado, las concepciones sobre investigación y, por otro, el proceso de supervisión en los programas de doctorado. Cada una de ellas tiene sus respectivas subcategorías y elementos asociados.

Tabla 3

Categorías emergentes

\begin{tabular}{l|l}
\hline $\begin{array}{l}\text { Concepciones sobre } \\
\text { investigación }\end{array}$ & $\begin{array}{l}\text { Supervisión en programas } \\
\text { de doctorado }\end{array}$ \\
\hline $\begin{array}{l}\text { Proceso de investigación } \\
\text { Importancia de la investigación }\end{array}$ & $\begin{array}{l}\text { Proceso de supervisión } \\
\text { Espacio educativo } \\
\text { Estrategias de investigación } \\
\text { Limitaciones/facilitadores }\end{array}$ \\
$\begin{array}{l}\text { Calidad en investigación } \\
\text { Vicios y malas prácticas investigativas del del estudiante } \\
\text { Perfil del investigador }\end{array}$ & $\begin{array}{l}\text { Fortalezas/facilitadores } \\
\text { Limitantes/obstaculizadores }\end{array}$ \\
\hline
\end{tabular}

Fuente: elaboración propia

En una tercera fase, los investigadores discutieron los criterios y categorías utilizadas para el análisis de datos, y posteriormente el uso de una grilla de análisis, en la cual se identificaron (1) categorías, (2) subcategorías, (3) discurso clave, (4) elementos claves e (5) interpretación. Un ejemplo de esta se puede ver en la tabla 4. 
Tabla 4

Grilla de análisis y categorización

\begin{tabular}{l|l|l|l}
\hline Categoría & Discurso claves & Elementos claves & Interpretación \\
\hline $\begin{array}{l}\text { Proceso de } \\
\text { investigación }\end{array}$ & $\begin{array}{l}\text { "O sea, a mí me parece que son competencias } \\
\text { que los estudiantes debiesen desarrollar, más } \\
\text { que por el resultado final, por la habilidad... } \\
\text { las habilidades durante el proceso de } \\
\text { investigar, ahí se desarrolla el pensamiento } \\
\text { crítico, reflexionar, eh, competencias mucho } \\
\text { más específicas (...) te ayuda a formular } \\
\text { preguntas relevantes". }\end{array}$ & $\begin{array}{l}\text { Pensamiento crítico. } \\
\text { Competencias } \\
\text { específicas. } \\
\text { Formulación de } \\
\text { preguntas. }\end{array}$ & $\begin{array}{l}\text { El proceso de investigación es } \\
\text { visualizado como una instancia } \\
\text { de desarrollo de habilidades de } \\
\text { pensamiento y competencias } \\
\text { específicas. Se destacan el } \\
\text { pensamiento crítico y la capacidad } \\
\text { de los investigadores de formular } \\
\text { preguntas relevantes y generar } \\
\text { reflexión frente a ella. }\end{array}$ \\
\hline \hline
\end{tabular}

Fuente: elaboración propia

Como criterios de confianza y validez, se utilizaron los principios propuestos por Yvonna S. Lincoln y Egon G. Guba (1985, citados por Mohanad Halaweh, Christine Fidler \& Steve McRobb, 2008): credibilidad, transferencia, confianza y adaptabilidad, al ser referidos como idóneos para investigaciones que involucren los dos métodos antes explicados. Para ello, desde la creación del guion de preguntas, el proceso de análisis, discusión y resultados fue sometido a dos momentos de triangulación; por un lado, la triangulación entre los investigadores y por otro, la triangulación entre los expertos y el marco teórico propuesto para esta investigación.

\section{Resultados}

Concepciones sobre investigación: la emergencia del conocimiento

Proceso de investigación

Las concepciones sobre investigación y sus dimensiones asociadas emergen como elementos transversales a la hora de entender la formación académica en los programas de posgrado. Esto obedece a la importancia que se da a la figura del supervisor, en su vertiente como investigador, docente y supervisor. En este sentido, los participantes evidencian la investigación como un ejercicio y necesidad fundamental, dinámica e inherente al quehacer humano. Permite la generación y la ampliación del conocimiento, y contribuye de esta forma al desarrollo de la comunidad.

La primera razón es la generación de conocimiento, todas las ciencias hacen esto, y lo... es eso, principalmente, de generar conocimiento que después puedan contribuir al desarrollo en muchos ámbitos de la sociedad, del humano ino cierto? (...) porque es algo inherente al ser humano. Yo creo que la historia entera de la humanidad ha mostrado esa necesidad del hombre de saber, de conocer más cosas (S3)'.

\section{Importancia de la investigación}

La investigación es visualizada como una instancia de desafío dinámico, en la que los saberes antiguos se confrontan con los nuevos. De esta forma, la investigación sirve como herramienta para renovar y cuestionar los paradigmas existentes, desarrollar habilidades de pensamiento y competencias específicas del investigador. Se destacan el pensamiento

1 magis transcribe las entrevistas con las marcas del lenguaje oral. magis no corrige ni edita las entrevistas. 
crítico y la capacidad de los investigadores de formular preguntas, obtener diferentes puntos de vistas frente a un problema y generar reflexión frente a él. Así, la investigación es capaz de identificar y completar vacíos existentes en el campo del conocimiento, para mejorar la calidad de vida de las personas por un lado y, por otro, para generar profesionales especialistas que puedan atender nuevos fenómenos o problemáticas emergentes en la disciplina.

Durante el proceso de investigar, se desarrolla el pensamiento crítico, reflexionar, eh, pero competencias — digamos - mucho más específicas (...) te ayuda a formular preguntas que sean relevantes, eh, poder argumentar, eh, por qué es relevante hacer un estudio o no, digamos, qué, qué impacto eso puede, puede tener, reflexionar en torno a lo que hay, y de qué manera lo que yo puedo investigar puedo tener un, un matiz diferente, una arista distinta (\$4).

Ahora bien, para que el investigador dentro de la Universidad pueda investigar, el discurso señala la necesidad de contar con preparación en el área de investigación; para ello, la formación en magíster y posgrados parece esencial:

Yo creo que el factor principal es la formación, primero de la persona que tiene que investigar, porque es diferente los conocimientos que tenga una persona que recién sale de la universidad a uno que ya tenga un grado académico de magíster o doctor (RDP).

\section{Estrategias de investigación}

Emergen a la vez dos estrategias para llevar a cabo la investigación. Por un lado, la actividad individual en la construcción del marco teórico y la problemática, para luego incorporar a otros colegas en el proyecto de investigación; la segunda estrategia consiste en la creación de grupos de investigación para un proyecto construido de forma colaborativa. Ambas estrategias son mencionadas como positivas; sin embargo, se evidencia la dificultad de trabajar en grupos de investigación, debido a las demandas presentes en la vida académica de los investigadores, fenómeno que se refleja en el siguiente discurso: "la posibilidad práctica de poder articularte con otros colegas de la misma unidad es, es difícil, lo intentamos, lo hacemos, pero, pero es un trabajo que tiende a ser también solitario" (S2).

\section{Limitaciones y facilitadores}

Entonces, la investigación es vista como una labor solitaria, pero a su vez demandante de interacción y formación de redes académicas y sociales. Esto permitiría no solo concretar y llevar a cabo el proceso de investigación, sino también darle visibilidad.

Uno de los principales obstáculos y limitantes presentes en este proceso se asocia a la multiplicidad de roles que deben cumplir los académicos en sus labores. Estas se visualizan desde tres áreas principales: docencia, investigación y vinculación con el medio. Estas áreas se consideran relevantes dentro de su labor, pero debido a la carga horaria dificultan el cumplimiento de los objetivos de investigación.

Yo creo que la universidad tiene una política en que todos tenemos que hacer de todo, y en ese, al menos en el ámbito académico y en ese afán es difícil como poder compatibilizar la investigación con la docencia, la gestión, y bueno vinculación con el medio (...) si bien dentro de la carga 
académica a uno le consideran horas para investigar (...) es difícil en la práctica articular eso con las exigencias cotidianas (...) por lo menos en lo personal casi es tiempo fuera del tiempo del trabajo, yo casi no trabajo investigación en mi horario de trabajo (S5).

Los recursos económicos y humanos son vistos como posibles obstáculos. Si bien son considerados determinantes en la labor investigativa, se menciona que esta puede llevarse a cabo, siempre y cuando haya condiciones de trabajo que permitan dedicar tiempos declarados para la investigación. Una de las soluciones planteadas por la mayoría de los participantes es una definición de roles académicos dentro de la Universidad que permita el perfil de investigador y docente puro.

Otro obstáculo destacado es la conceptualización de la empresa privada como un agente durmiente en el proceso de la investigación científica chilena. Es vista como un agente relevante en el ámbito internacional, pero que actualmente, en el país, no hay instancias o propuestas en las cuales la empresa privada participe y aporte para la creación de conocimiento.

Yo soy un crítico, veo las realidades extranjeras ino cierto? La cantidad de dinero que invierte la empresa privada en la investigación (...) el aporte de la empresa privada es lo que promueve la investigación (cambio en tono de voz) científica y aplicada. Aquí, la verdad es que no hemos logrado como país motivar a la empresa u... u... obligar a la empresa (risas) que, de alguna forma, invierta en investigación (S6).

Al referirse a los facilitadores de este proceso, los entrevistados señalan la motivación del propio investigador como uno de los elementos clave. Esta característica es concebida como profundamente personal y propia del quehacer académico. Si bien hay incentivos por parte de la universidad, estos son percibidos como insuficientes en comparación entre las áreas de investigación y otras instituciones educativas. Se destaca también la facilidad que brinda la universidad para investigar en áreas de interés del investigador y la disposición para perfeccionamiento y capacitación.

A pesar de toda la carga académica, igual la universidad nos da como hartas posibilidades de investigar, por ejemplo, investigamos solo temas que nosotros queremos (...), tener eh, la oportunidad de investigar en lo que nosotros queramos, podríamos tener restricciones por ser una universidad católica, nada, acá nosotros investigamos en lo que queramos (...) siempre está la disposición, o sea, la buena voluntad (...), en general, nos intentan facilitar más la vida (S3).

\section{Calidad en investigación}

Al hablar sobre las características de una investigación de calidad, los entrevistados señalan que una buena investigación debe estar asociada a una correcta búsqueda de información que sustente el problema y la temática a investigar. Se debe tener conocimiento de los procedimientos y del orden necesarios. Destacan la coherencia entre los niveles ontológicos, epistemológicos y metodológicos de la investigación.

La rigurosidad por ejemplo en la búsqueda de información, eh, la rigurosidad respecto de las afirmaciones que se hacen, eh, cuando tú estás argumentando una, una pregunta o un problema de investigación, eh, la rigurosidad también me refiero a los aspectos éticos involucrados, eh, sobre todo cuando tú trabajas con personas ino? ya sea con el resguardo de 
las cuestiones que tiene qué ver con no dañar, con proteger el bienestar de las personas, pero también los resguardos éticos que tienen qué ver con el manejo de los datos (S4).

Vicios y malas prácticas investigativas

Si bien se comparten los criterios asociados a la investigación, los estudiantes de doctorado no hacen referencia a los aspectos éticos involucrados en los procesos de investigación. De esta forma, reducen desde el discurso, la investigación a aspectos formales y técnicos, en los que el fantasma de la focalización en la producción científica (Brooks \& Heiland, 2007; Deem, 2006) parece tomar fuerza.

Algunas cosas éticas no se consideran, yo creo que más por desconocimiento que por una cosa, como por algo ex profeso de... de... de transgredir una norma, creo que no se (ruido externo). Quizás ahí es una responsabilidad de nosotros los formadores como de... de (ruido externo) poner más hincapié en..., que seguir ciertos procedimientos, que hay que eva... evaluar el daño, qué pasa si yo abro ciertos temas, cómo los cierro, eh, que a veces esas cosas se dejan un poco de lado y, claro... ahí cometes algunos errores en ese ámbito (S3).

Esta excesiva focalización en la producción científica — representada en la publicación indexada de la investigación - permite la aparición de vicios o malas prácticas en los procesos de investigación. Estos son vistos como lejanos y distantes a la realidad local. Por ejemplo, el proceso de publicación es percibido como un evento estresante para el investigador. La publicación indexada se entiende como el culmen de los procesos de investigación, pero a su vez como una presión para el investigador. Se explica así, que esta presión genera competencia ante el requerimiento de cumplir cuotas de publicación. Aparecen entonces conductas o actividades no comprendidas en la realidad local, pero sí explicadas desde estas presiones, como el acuerdo entre investigadores para incluirse mutuamente en sus investigaciones y rumores de pagos para publicaciones. Otra práctica vista es el mal uso de las herramientas metodológicas para explicar resultados, ya sea por desconocimiento, poca experticia en el área, o por reflejar resultados de forma diferente.

Entonces, siempre hay una presión grande, y por... y... y el sistema mismo no... nos lleva mucho a eso, eh, casi no tenemos mucho tiempo para pensar bien las cosas, queremos hacerlo rápido, porque rápido tengo que publicar, un proyecto FONDECYT² de tres años, uno ya en el primer año tiene que mostrar alguna publicación y eso es bastante presionante (D1).

\section{Perfil del investigador}

Del discurso de los participantes, emerge así que el perfil del buen investigador es subjetivo, y que hay una serie de características profesionales y personales diversas. Sin embargo, algunos elementos se mencionan de forma clara. Por un lado, las habilidades de pensamiento superior que pueden favorecerlo en su ejercicio como investigador, tales como el

2 El Fondo Nacional de Desarrollo Científico y Tecnológico, FONDECYT, es el principal fondo público del Gobierno de Chile, dependiente de la Comisión Nacional de Investigación Científica y Tecnológica, CONICYT, para incentivar la investigación científica en todas las áreas del conocimiento. Abierto en 1984, hasta 2017 había financiado más de 13.500 proyectos de investigación con la participación de más de 12.000 investigadores. 
pensamiento crítico, la capacidad de aprendizaje continuo, el nivel intelectual y la motivación por la investigación. Estas características se condicen con lo planteado por Manuel Krauskopf (2003), quien refiere que estas habilidades emergen producto de la formación en los programas de doctorado. Se mencionan también características asociadas a la rigurosidad y proactividad en los procesos de investigación.

Lo que veo en común entre las personas con las que me toca trabajar en investigación es esa capacidad de síntesis, capacidad y claridad para entender conceptos, integrar conceptos; yo creo que esa es la parte esencial, independiente de... del comportamiento (S5).

Hay diferencias según la disciplina de investigación en cuanto a estas características; por ejemplo, en las áreas de ciencias duras, se destacan la capacidad y el nivel intelectual del investigador, mientras que en las ciencias aplicadas y blandas, los aspectos asociados a la motivación, la disposición y el interés por la investigación.

Si bien el perfil es identificado como subjetivo, los estándares nacionales de medición están fuertemente ligados al índice de publicación del investigador y a la obtención de fondos para investigar. Es decir, por un lado, se destaca el perfil del investigador con una serie de elementos y características personales y profesionales y, por otro, se evidencia que la medición real del investigador sigue siendo un elemento cuantitativo otorgado por los índices de publicación obtenidos.

Supervisión en los programas de doctorado: la formación de futuros investigadores

\section{Proceso de supervisión}

Este es visualizado como una instancia que debe comenzar tempranamente, al demostrar compromiso por parte del supervisor con el estudiante. Este compromiso se traduce no solo en la evaluación del avance del estudiante, sino también en mantener un proceso en el cual compartan espacios para discutir y reflexionar sobre los avances realizados. De esta forma, el proceso de supervisión se transforma en una relación dinámica y dialéctica entre el estudiante y el supervisor, para que ambos puedan avanzar en el aprendizaje.

Emergen a la vez dificultades en las cuales el supervisor - por sobrecarga académica - no es capaz de responder a este compromiso. Esto se evidencia en retroalimentaciones someras, por cumplimiento con el estudiante y no por el compromiso contraído con el proceso de supervisión. A mediano plazo, esta situación termina por afectar el cumplimiento de los plazos establecidos por el programa de posgrado.
(...) falta de compromiso de los profesores guía, o sea, de pronto, el doctorando no, no en todos los casos, pero en la mayoría de los casos, el doctorando queda un poco aislado, solitario. Digamos... parte de nuestro primer año, en nuestro... en nuestro programa, con los cursos y diseñando su propuesta (cambio en tono de voz) y el profesor ahí como que lo apoya un poco, luego vienen dos años de investigación en que si el profesor no está directamente involucrado... lo deja como de lado hasta que ya empieza el cuarto año y empieza a apretar para que la publicación, que la tesis, y toda la historia (\$4).

\section{Espacio educativo}

La supervisión es vista como una instancia pedagógica que plantea desafíos tanto al supervisor como al estudiante pues se deben considerar las particularidades de ambos, para crear espacios de interacción y aprendizaje necesarios para la formación de los futuros investigadores.

\section{Roly perfil del supervisor}

En cuanto al rol y perfil del supervisor, se establece como un guía que debe orientar y aconsejar al estudiante durante su proceso de posgrado. Si bien se evidencia que el estudiante debe ser más autónomo, también se destaca el compromiso del supervisor para involucrarse en el trabajo de su estudiante. También se evidencia que debido a debilidades de los estudiantes - en especial asociadas con la redacción científica-, en ocasiones, el supervisor debe tomar un rol mucho más activo en el trabajo del docente. Esto ocasiona que la participación del supervisor en la tesis del estudiante sea más notoria y que, en algunos casos, termine de redactar grandes partes de ella.

Los entrevistados señalan el desconocimiento de la universidad sobre el rol que cumple el académico como supervisor en estas instancias. Si bien se visualizan los posibles beneficios de apoyar las investigaciones de los estudiantes de posgrado, la universidad no percibe el trabajo realizado, pues asimila el tiempo dedicado a una tesis de pregrado con el de una tesis de posgrado.

La misión del profesor guía no se reconoce en la intensidad que debería, o sea, llevar una tesis doctoral con propiedad ino cierto?, no es lo mismo que llevar una tesis de pregrado. Y básicamente nuestro ordenamiento, eh, institucional es prácticamente... y se reconoce casi con la misma intensidad que dirigir una tesis de pregrado que puede tomar seis meses ¿no cierto? una cuestión muy liviana... en desarrollar ciencia profunda en un... en una... doctorando en que tienes que invertir mucho más tiempo, mucha más energía, eh, muchas horas de trabajo (56). 
En el discurso, emerge una distinción en algunos de los supervisores y estudiantes frente a los elementos centrales del supervisor. Se defiende la idea del supervisor como mentor, con herramientas pedagógicas y personales, y se involucra con el quehacer del estudiante, como se refleja en el siguiente discurso:

Que se involucre contigo como persona, o sea en el fondo tú estás trabajando con él cuatro años mínimo, o sea, mínimo que sepa... eh, tu nombre, dónde vives, si tienes un hijo, cómo se llama tu hijo, ¿me entiendes? o sea, que tenga esa..., ese querer ser parte de..., no solo de la investigación, sino que de la vida que uno lleva (E7).

Sin embargo, también emerge la figura del supervisor como eminencia, cuya principal característica debe ser su capacidad de conocer y comprender la temática que está trabajando. Si bien se identifica la necesidad de ser cercano con el estudiante, no es una característica determinante, como sí lo es el nivel de conocimiento del supervisor y su preocupación por el avance del estudiante. Aun cuando se identifican características personales y profesionales que facilitan el proceso de supervisión, estas son subjetivas y no visualizadas como elementos incidentes en el desempeño del supervisor. En otras palabras, si el supervisor es conocedor de la temática y si está comprometido con la labor realizada por el estudiante, las características personales del supervisor pasan a segundo plano.

Hay casos en que el profe no es muy simpático pero es tremendamente aportador, el profe es estricto, es serio, no es muy llano a compartir, ino cierto? pero es tremendamente aportador. Yo creo que lo importante es que exista el compromiso, independiente de las cualidades personales del investigador, si hay un compromiso y hay una claridad en él, en la investigación que se está realizando por parte del profe guía, yo creo [que] eso soluciona cualquier problema (S3).

\section{Roly perfil del estudiante}

En cuanto al perfil del estudiante de posgrado, este se construye como un estudiante que debe tener conocimiento base sobre el área en la cual desea investigar. Se evidencian unas características necesarias para el cumplimiento de los objetivos propuestos por los programas, como proactividad, capacidad cognitiva, autonomía e independencia, pensamiento crítico y reflexivo, tolerancia a la frustración, responsabilidad y organización. Si bien se enumeran una serie de características asociadas al perfil del estudiante, los supervisores son críticos con respecto a ellos. Se indica que muchos no son capaces de desarrollar autonomía en su labor, pues aún dependen mucho de la figura del supervisor para la toma de decisiones. Esta falta de autonomía se evidencia desde el pregrado, ya sea por inseguridad o por la dependencia del estudiante de las figuras docentes. También destacan las dificultades que los estudiantes presentan en cuanto a redacción y desarrollo de ideas, y las complicaciones con la comprensión y el desempeño en el idioma inglés, considerado como el lenguaje esencial para la investigación.

Ingresar a un programa de doctorado y, por ende, aceptar el desafío de dedicarse a la academia y a la investigación es un acto muy personal y con componentes motivacionales. Esta es una característica deseable en los estudiantes, tal cual refleja el siguiente discurso: "le tiene que gustar la investigación, yo creo pa' estar en esto te tiene que gustar, o sea, tienes que amarlo (risa); si no, es superdifícil mantenerte en esto" (S3). 
En este punto, emergen algunas diferencias entre el discurso de supervisores y estudiantes. Mientras los supervisores ven en el perfil del estudiante características cognitivas necesarias, orden y capacidad de reflexión, los estudiantes refieren que si bien estos elementos son fundamentales, la motivación, el interés y lo que puede ser llamado como el romance con la investigación son necesarios. Joseph S. Renzulli, Jennifer L. Koehler y Elizabeth A. Fogarty (2006) utilizan el concepto romance con el tópico o la disciplina, al referirse a una pasión intrínseca e individual en las personas, caracterizada por fuertes emociones y deseos en relación con el tema, este fenómeno termina por cautivar y comprometer a las futuras generaciones. En este caso, a los investigadores en formación.

... responsable, eh, que sepa trabajar en equipo, que sea perseverante, yo creo para hacer un doctorado, no necesita ser un supergenio ni tener un $\mathrm{Cl}$ muy alto, o sea, tienes que tener las ganas de que... $\mathrm{y} \ldots \mathrm{y}$, de querer hacer eso que te gusta y hacerlo bien (E4).
Fortalezas/facilitadores

Se visualizan fortalezas y desventajas que los estudiantes pueden enfrentar. Se menciona la motivación, pasión y elección de la vida académica como un agente esencial en ellos. Se toman en consideración las dificultades que los propios supervisores declaran que tiene la vida académica y se considera esta elección como una decisión de vida.

... pero levantarse todos los días temprano a hacer algo que no me gusta, o sea, no, prefiero no, paro ahí, pero lo que yo he visto en ellos, en todos, es que lo que hacen... su área les gusta, el..., les apasiona, lo hacen con dedicación (S5).

\section{Limitantes/obstáculos}

En el caso de las desventajas y obstáculos, se menciona principalmente el financiamiento que los estudiantes deben tener para dedicarse a los estudios; la gestión del tiempo; el excesivo trabajo individual y ciertas conductas de entrada, específicamente el manejo del inglés y la lectoescritura científica.

Gráfico 1

Matriz condicionada sobre la formación en investigación y la supervisión en programas de doctorado

Formación en investigación y supervisión

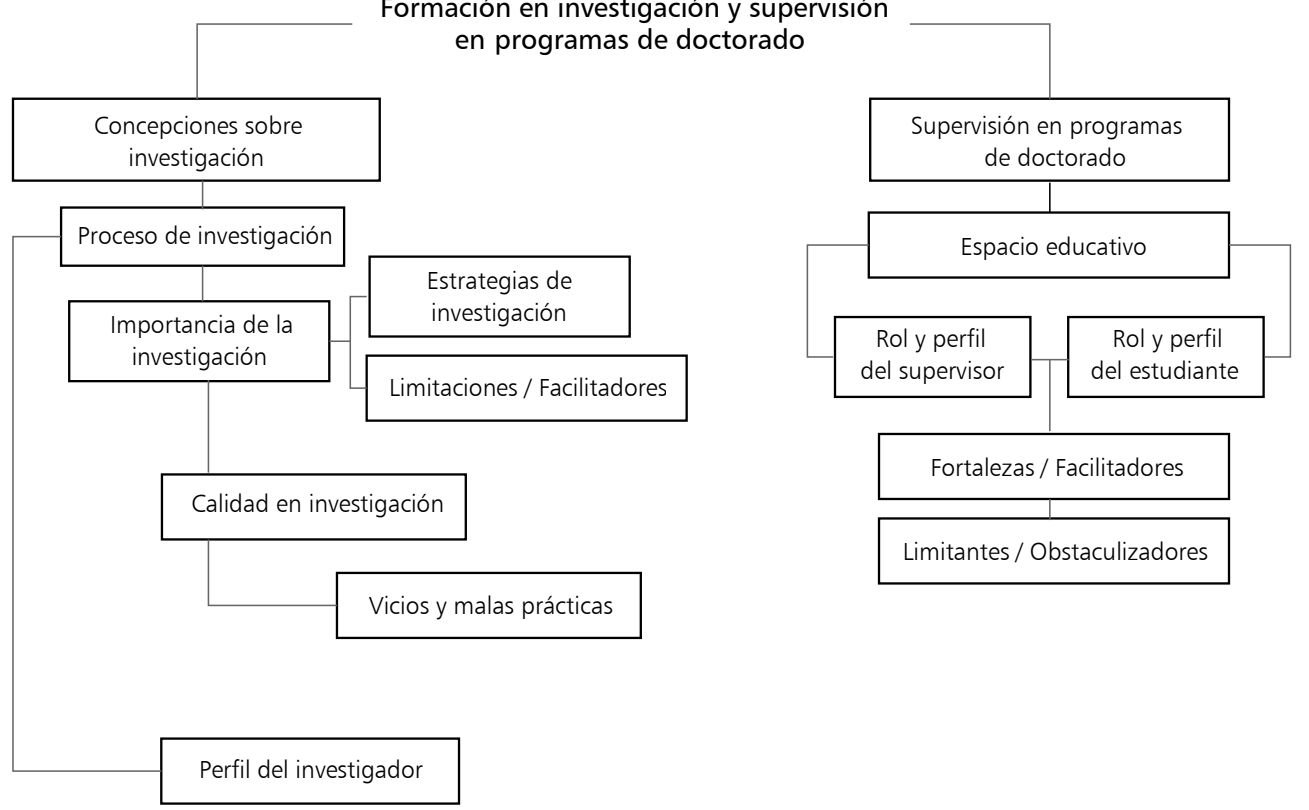

Fuente: elaboración propia 


\section{Discusión y conclusión}

Dos grandes categorías emergen de esta investigación: las concepciones que se tienen sobre los procesos de investigación, y la supervisión de los programas de doctorado. El gráfico 1 representa mediante el modelo teórico obtenido de la matriz selectiva, cómo ambas dimensiones interactúan entre sí. Estas se entienden como procesos que van de la mano, en los cuales la formación en investigación del supervisor incide directamente en cómo se forma a los nuevos investigadores. Esto se relaciona con la idea propuesta por Anita Kärner y Väino Puura (2008), en la cual la formación de posgrado es vista como un espacio fértil para el desarrollo de las competencias necesarias para la investigación. Se permite así al estudiante conocer el marco científico regulador, labor que recae en el supervisor desde su rol como experto y sujeto partícipe de la comunidad científica. Durante el proceso de formación, el supervisor entrega herramientas que le permitan al estudiante conocer los estándares esperados y las demandas del entorno, como lo refieren Renske A. M. Kleijn, M. Tim Mainhard, Paulien C. Meijer, Albert Pilot y Mieke Brekelmans (2010). Ahora bien, estas herramientas y conocimientos corresponden a la cultura académica desde la cual se sitúa el supervisor. Como menciona Tony Becher (1993), estas culturas en ocasiones son inmutables y con el tiempo, se cristalizan, lo que impide su flexibilidad y evolución. Esta reflexión permitiría conocer de mejor forma los procesos de formación de los futuros investigadores dentro de la universidad.

En cuanto a la supervisión de los programas de doctorado, la figura del supervisor se destaca como altamente demandante por su entorno. Para Ross Deuchar (2008), el investigador no solamente es evaluado como tal, sino también como supervisor, docente y agente de vinculación con el medio. Esta situación genera conflicto, pues la labor investigativa dentro de la universidad se percibe como solitaria, lo que permea los procesos de supervisión, como afirman Ronny K. Gunnarsson, Grethe Jonasson y Annika Billhult (2013).

Hay una fuerte demanda del entorno, por la producción científica, como sostienen Rachelle L. Brooks y Donna Heiland (2007) y Rosemary Deem (2006). Esta focalización permea los procesos de investigación dentro de la Universidad y, a su vez, en la formación de los futuros investigadores. Esta situación se convierte en una presión hacia los investigadores, que evidencian de forma lejana, pero real, malas prácticas investigativas para cumplir estos indicadores.

En cuanto al rol del supervisor, los resultados concuerdan con lo planteado por Christine Bruce e lan Stoodley (2013), Ross Deuchar (2008), Margaret Kiley (2011), Liina Lepp, Marvi Remmik, Mari Karm y Äli Leijen (2013), Margot Pearson y Carole Kayrooz (2004) y Stan Taylor y Nigel Beasley (2005), respecto a su importancia. Sin embargo, hay diferencias en cuanto a las características más relevantes. Por un lado, los estudiantes señalan la necesidad del involucramiento personal del supervisor y, por otro, los supervisores mencionan que la experticia del investigador y su compromiso son mejores predictores para la formación de los estudiantes que las características personales atribuidas por estos.

Al referirse a los facilitadores, la motivación y compromiso hacia la investigación se destacan como claves para la carrera académica, que se considera como un ejercicio y una decisión de vida. Este fenómeno implica al investigador personal, profesional y emocionalmente, si se considera el aporte de su labor al campo del conocimiento de la disciplina. 
En términos generales, los resultados presentados en esta investigación reflejan una realidad compartida de acuerdo a la teoría revisada. Las percepciones y concepciones que se tienen sobre la investigación por parte de estudiantes y supervisores — si bien presentan particularidades propias del contexto- comparten lineamientos comunes asociados al marco científico que rodea los procesos de investigación. La formación de investigadores demanda el desarrollo de competencias metacognitivas, supeditadas al desarrollo de habilidades formales para acceder al objetivo implícito, actual y final de la investigación: la publicación indexada. Por esta razón, habilidades de redacción científica y el dominio del idioma inglés son vistos como herramientas esenciales para lograr estos objetivos. La aparición de esta meta, tanto implícita como explícita desde el discurso de los entrevistados, se configura como un elemento construido y perpetuado en la cultura académica de la Universidad Católica del Norte.

Si bien los tres programas de doctorado son concebidos desde diferentes disciplinas, el discurso central en torno a la investigación parece no tener diferencias. Esto puede explicarse en función de lo planteado por Rachelle L. Brooks y Donna Heiland (2007) y Rosemary Deem (2006), según lo cual la focalización en la producción científica parece estar permeando las concepciones sobre investigación y la formación de los futuros investigadores. Un único elemento emerge como diferencial entre los programas: el rol afectivo y de contención del supervisor en el proceso de formación de investigadores. Este rol es una característica esperable y deseable en el programa de psicología, demandada y evidenciada por estudiante y supervisor; en el programa de ciencias mención geología, esta es más esperable y deseable por el estudiante en el supervisor; mientras que no parece ser un elemento relevante en el proceso para ninguno de los involucrados en el programa de ciencias mención matemáticas.

En conclusión y de acuerdo a los resultados y la discusión propuesta, la formación de futuros investigadores en los programas de doctorado de la Universidad Católica del Norte es visualizada como una relación solitaria y de formación educativa, en la cual la figura del supervisor es vista en función de sus características personales y profesionales. El estudiante busca en esta figura mayor cercanía e identifica elementos profesionales y emocionales imprescindibles para el éxito de esta relación pedagógica. A su vez, hay demandas de ambos agentes hacia la Universidad, a fin de validar este espacio educativo como una instancia académica esencial en la labor universitaria. En la Universidad Católica del Norte, la investigación es visualizada como una acción romántica, en la cual tanto el supervisor como el investigador en potencia deben establecer un lazo motivacional con una fuerte carga emocional hacia la labor investigativa. Finalmente, hay que visualizar el rol regulador existente desde la formación en investigación; aquellos aspectos cultural y académicamente inmersos de manera implícita que forman al investigador bajo un marco de reglas preestablecidas e inherentemente aceptadas por la comunidad científica, como lo menciona Tony Becher (1993). Así, el supervisor se convierte en un agente educativo y formativo de los nuevos investigadores con la responsabilidad de traspasar efectivamente aquellos conocimientos explícitos e implícitos que conlleva la acción científica.

Los resultados de esta investigación muestran que la supervisión es un proceso en el que convergen distintos elementos que van configurando diversas trayectorias. En este sentido, haber entrevistado solo a estudiantes que cursan los programas de doctorado, sin considerar a los estudiantes desertados, constituye una posible limitación de este estudio. Profundizar en aquellos estudiantes que no hayan seguido con este proceso formativo, 
podría brindar luces sobre elementos no contemplados en esta investigación. Otra de las limitaciones fue el trabajo como estudio de caso al considerar solamente el campus Antofagasta de la Universidad Católica del Norte. Las distancias geográficas no permitieron realizar el estudio de campo con el resto de los programas de doctorado en Coquimbo y San Pedro de Atacama.

Resultados como los reportados en esta investigación permiten comprender la complejidad asociada a la formación de investigadores y la dinámica existente en los procesos de supervisión. Generar espacios formales de discusión y reflexión entre investigadores, universidad y estudiantes parece ser el camino para romper la estructura solitaria de estos procesos. Futuras investigaciones deben profundizar en aquellas competencias y habilidades necesarias en los futuros investigadores, y en las repercusiones e implicancias de los contenidos implícitos y cómo las culturas académicas en los programas de doctorado de la Universidad se hacen cargo de ellos.

\section{Sobre los autores}

Alejandro Proestakis-Maturana es subdirector académico del Centro de Investigación y Desarrollo de Talentos, Universidad Católica del Norte. Docente y supervisor de tesis para las escuelas de pedagogía y psicología, Universidad Católica del Norte. Investigador en áreas asociadas a educación, talento y creatividad.

Walter Terrazas-Núñez es decano de la Facultad de Humanidades, Universidad Católica del Norte. Docente en cursos de pregrado y posgrado de la escuela de psicología. Investigador en área de enseñanza y aprendizaje en educación superior.

\section{Referencias}

Armstrong, Steven J.; Allinson, Christopher W. \& Hayes, John (2004). The Effects of Cognitive Style on Research Supervision: A Study of Student-Supervisor Dyads in Management Education. Academy of Management Learning \& Education, 3 (1), 41-63. Disponible en: http:// www.jstor.org/stable/40214230

Becher, Tony (1993). Las disciplinas y la identidad de los académicos. Pensamiento Universitario, 1 (1). 56-77. Disponible en: http://inter27.unsl. edu. ar/rapes/?action $=$ detalle\&from $=$ todos\&id $=441$

Berg, Bruce L. (2001). Qualitative Research Methods for the Social Sciences. $4^{\text {th }}$ ed. Boston, Massachusetts: Allyn \& Bacon.

Boud, David \& Lee, Alison (eds.) (2008). Changing Practices of Doctoral Education. London: Routledge.

Braun, Virginia \& Clarke, Victoria (2006). Using Thematic Analysis in Psychology. Qualitative Research in Psychology, 3 (2), 77-101. DOI: 10.1191/1478088706qp063oa

Brew, Angela (2001). Conceptions of Research: A Phenomenographic Study. Studies in Higher Education, 26 (3), 271-285. DOI: 10.1080/03075070120076255

Brooks, Rachelle L. \& Heiland, Donna (2007). Accountability, Assessment and Doctoral Education: Recommendations for Moving Forward. European Journal of Education, 42 (3), 351-362. DOI: 10.1111/j.14653435.2007.00311.x

Bruce, Christine \& Stoodley, Ian (2013) Experiencing Higher Degree Research Supervision as Teaching. Studies in Higher Education, 38 (2), 226-241. DOI: 10.1080/03075079.2011.576338

Bruce, Christine; Stoodley, Ian \& Pham, Binh (2009). Doctoral Student's Experience of Information Technology Research. Studies in Higher 
Education, 34 (2), 203-222. Disponible en: http://www.chrismills.com. au/files/335PDF/doctoral\%20student\%20technology\%20research.pdf Council of Graduate Schools, CGS, PhD Completion Program (2010). PhD Completion and Attrition: Findings from Exit Surveys of PhD Completers. Monograph. Released Sept. 2009.

Deem, Rosemary (2006). Conceptions of Contemporary European Universities: To Do Research Or Not To Do Research? European Journal of Education, 41 (2), 281-304. DOI: 10.1111/j.1465-3435.2006.00260.x

Deuchar, Ross (2008). Facilitator, Director or Critical Friend?: Contradiction and Congruence in Doctoral Supervision Styles. Teaching in Higher Education, 13 (4), 489-500. DOI: 10.1080/13562510802193905

Edwards, Brian (2002). Postgraduate Supervision: Is Having a PhD Enough? A paper presented at the Australian Association for Research in Education Conference Problematic Futures: Educational Research in an Era of... Uncertainty. Brisbane, Australia 1- 5, December, 2002. Disponible en: https://www.aare.edu.au/data/publications/2002/ edw02382.pdf

Gaeta, Giuseppe Lucio (2013). Was it Worth It?: An Empirical Analysis of Over-Education among PhD Recipients in Italy. c.MET Working paper 02/2013. Disponible en: http://193.205.129.80/repec/cme/wpaper/ cmetwp_02_2013.pdf

Gardner, Susan K. (2009). Student and Faculty Attributions of Attrition in High and Low-Completing Doctoral Programs in the United States. Higher Education, 58 (1), 97-112. DOI: 10.1007/s10734 008-9184-7

Green, Bill \& Lee, Alison (1999). Educational Research, Disciplinarity and Postgraduate Pedagogy: On the Subject of Supervision. Review of Australian Research in Education, 5, 95-111.

Gunnarsson, Ronny K.; Jonasson, Grethe \& Billhult, Annika (2013). The Experience of Disagreement between Students and Supervisors in PhD Education: A Qualitative Study. BioMedCentral, BMC Medical Education, 13, 134. DOI: 10.1186/1472-6920-13-134. Disponible en: https://www.researchgate.net/publication/257202573_The_expe rience_of_disagreement_between_students_and_supervisors_in PhD_education_A_qualitative_study

Halaweh, Mohanad; Fidler, Christine \& McRobb, Steve (2008). Integrating the Grounded Theory Method and Case Study Research Methodology within IS Research: A Possible Road Map. En ICIS 2008 Proceedings, Twenty Ninth International Conference on Information Systems, Paris France, 14-17 December 2008, 242-253. Atlanta, Georgia: International Conference on Information Systems.

Kärner, Anita \& Puura, Väino (2008). Doctoral Education in Transition to Knowledge-based Society. Trames, 12 (1), 95-109. DOI: 10.3176/ tr.2008.1.06. Disponible en: http://www.kirj.ee/public/trames_ pdf/2008/issue_1/trames-2008-1-6.pdf

Kehm, Barbara M. (2006). Doctoral Education in Europe and North America: A Comparative Analysis. En Ulrich Teichler (ed.). The Formative Years of Scholars, 67-78. Portland Press: London, Series WennerGren International Series (83). Disponible en: http://www.portlan dpress.com/pp/books/online/fyos/083/0067/0830067.pdf, http:// www.portlandpress.com/pp/books/online/fyos/083/default.htm

Kiley, Margaret (2011). Developments in Research Supervisor Training: Causes and Responses. Studies in Higher Education, 36 (5), 585-599. DOI: 10.1080/03075079.2011.594595

Kiley, Margaret \& Wisker, Gina (2009). Threshold Concepts in Research Education and Evidence of Threshold Crossing. Higher Education 
Research and Development, 28 (4), 431-441. DOI: 10.1080/072943 60903067930

Kleijn, Renske A. M.; Mainhard, M. Tim; Meijer, Paulien C.; Pilot, Albert \& Brekelmans, Mieke (2010). Master's Thesis Supervision: Relations between Perceptions of the Supervisor-Student Relationship, Final Grade, Perceived Supervisor Contribution to Learning and Student Satisfaction. Studies in Higher Education, 37 (8), 925-939. DOI: 10.1080/03075079.2011.556717

Krauskopf, Manuel (2003). Indicadores cuantitativos de los doctorados conferidos en el país. ¿Falta de atención o expresión de subdesarrollo? Revista Calidad en la Educación, 18, 47-59. Disponible en: https://www.researchgate.net/publication/267789804_INDICA DORES_CUANTITATIVOS_DE_LOS_DOCTORADOS_CONFERIDOS EN_EL_PAIS_FALTA_DE_ATENCCION_O_EXPRESIŌN_DE_SUBDE SARROLLO

Lepp, Liina; Remmik, Marvi; Karm, Mari \& Leijen, Äli (2013). Supervisor' Conceptions of Doctoral Studies. Trames, 17 (4), 401-415. DOI: 10.3176/tr.2013.4.06. Disponible en: http://www.kirj.ee/public/tra mes_pdf/2013/issue_4/Trames-2013-4-401-415.pdf

Lincoln, Yvonna S. \& Guba, Egon G. (1985). Naturalistic Inquiry. Beverly Hills, California: Sage.

Litalien, David \& Guay, Frédéric (2015). Dropout Intentions in PhD Studies: A Comprehensive Model Bases on Interpersonal Relationships and Motivational Resources. Contemporary Educational Psychology, 41, 218-231. DOI: http://dx.doi.org/10.1016/j.cedpsych.2015.03.004

Manathunga, Catherine; Lant, Paul \& Mellick, George (2006). Imagining an Interdisciplinary Doctoral Pedagogy. Teaching in Higher Education, 11 (3), 365-379. DOI: 10.1080/13562510600680954

McAlpine, Lynn \& Norton, Judith (2006). Reframing Our Approach to Doctoral Programs: An Integrative Framework for Action and Research. Higher Education Research and Development, 25 (1), 3-17. DOI: 10.1080/07294360500453012

Nettles, Michael T. \& Millett, Catherine M. (2006). Three Magic Letters: Getting to PhD. Baltimore, Maryland: Johns Hopkins University Press.

Nulty, Duncan; Kiley, Margaret \& Meyers, Noel (2009). Promoting and Recognising Excellence in the Supervision of Research Students: An Evidence-Based Framework. Assessment \& Evaluation in Higher Education, 34 (6). DOI: 10.1080/02602930802474193

Organisation for Economic Co-operation and Development, OECD (2013). Students Enrolled by Type of Institution [Statistic tables]. Disponible en: http://stats.oecd.org

Pearson, Margot \& Kayrooz, Carole (2004). Enabling Critical Reflection on Research Supervisory Practice. The International Journal for Academic Development, 9 (1), 99-116. DOI: 10.1080/1360144042000296107

Renzulli, Joseph S.; Koehler, Jennifer L. \& Fogarty, Elizabeth A. (2006). Operation Houndstooth Intervention Theory: Social Capital in Today's Schools. Gifted Child Today, 29 (1), 14-24. Disponible en: http://gif ted.uconn.edu/wp-content/uploads/sites/961/2015/09/Social_Capi tal_in_Todays_Schools.pdf

Sadlak, Jan (2004). Doctoral Studies and Qualifications in Europe and the United States: Status and Prospects. Bucharest: United Nations Educational, Scientific and Cultural Organization, Centre Européen pour l'Enseignement Supérieur/European Centre for Higher Education, UNESCO-CEPES. Disponible en: http://unesdoc.unesco.org/ images/0013/001364/136456e.pdf 
Schreier, Margrit (2012). Qualitative Content Analysis in Practice. Thousand Oaks, California: Sage.

Taylor, Stan \& Beasley, Nigel (2005). A Handbook for Doctoral Supervisors. London: Routledge Falmer.

Titus, Sandra L. \& Ballou, Janice M. (2013). Ensuring PhD Development of Responsible Conduct of Research Behaviors: Who's Responsible? Science and Engineering Ethics, 20 (1), 221-235. DOI: 10.1007/ s11948-013-9437-4

Wright, April; Murray, Jane P. \& Geale, Patricia (2007). A Phenomenographic Study of What it Means to Supervise Doctoral Students. Academy of Management Learning and Education, 6 (4), 458-474. DOI: 10.5465/amle.2007.27694946 